\title{
Specific dynamic of serum procalcitonin in critically ill patients affected by Gram-negative bacilli septic thrombophlebitis
}

\author{
Martina Spaziante ${ }^{1,2}$, Giancarlo Ceccarelli, ${ }^{1,2}$, Samir Al Moghazi ${ }^{1,2}$, Francesco Alessandri ${ }^{2,3}$ \\ and Mario Venditti ${ }^{1,2^{*}}$
}

We read with interest the study by Thomas-Rüddel et al. [1] evaluating the influence of specific pathogens and different foci of infections on serum procalcitonin (PCT) concentrations. The authors concluded that PCT levels were higher in patients with Gram-negative bacteremia compared with patients with Gram-positive or fungal diseases, whereas urogenital and abdominal foci of infection were associated with twofold increased PCT values, independent of causative pathogen. Unfortunately, this study did not provide data on PCT trends in patients affected by endovascular infections.

We recently collected a small series of 13 cases of endovascular infections caused by thrombophlebitis due to Gram-negative bacilli (GNB) in the intensive care unit (ICU) of a large University Hospital in Italy. The mean age of patients enrolled was $59.2 \pm$ 13.6 years with a predominance of male sex (61.5\%); the mean SAPS II at the admission was $39.7 \pm 8.1$ points and the most frequent cause of ICU admission was a recent polytrauma (84.6\%). All patients had persistent bacteremia despite administration of in vitro active antibiotics and removal of intravascular devices. The diagnosis of septic thrombophlebitis was corroborated by CT scan (53.8\%) or echodoppler (46.2\%), and thrombus appositions mainly involved aortic trunks (61.5\%). The blood isolates were four Klebsiella pneumoniae, four Acinetobacter baumannii, one Enterobacter spp., one Pseudomonas aeruginosa, one Morganella morganii, one Providencia rettgeri, and one Klebsiella oxytoca.

Despite the prolonged duration of bacteremia and the appropriate antibiotic therapy, all patients showed an indolent clinical course, with no multi-organ failure, prompt clinical improvement, and rapid decrease of plasma PCT concentrations within normal ranges after the onset of septic episodes (Fig. 1).

As previously reported, PCT is produced in response to inflammatory cytokines and bacterial endotoxins [2]. In our cases the rapid decrease of PCT, followed by a stable normalization of serum concentration despite persistence of bacteremia, could be explained with the well-known mechanism of immune tolerance: in fact the selective blocks of some pro-inflammatory pathways, activated by bacterial endotoxins or cytokines, could impact on the production of PCT and favor a long indolent clinical course, even in the face of microbial eradication failure [3].

In conclusion, we think that our data could contribute to complete the results of Thomas-Rüddel et al. and are worthy of being further investigated in a

* Correspondence: mario.venditti@uniroma1.it

${ }^{1}$ Department of Public Health and Infectious Diseases, University of Rome

Sapienza, Viale del Policlinico 155, Rome, Italy

${ }^{2}$ Azienda Policlinico Umberto I, Rome, Italy

Full list of author information is available at the end of the article 


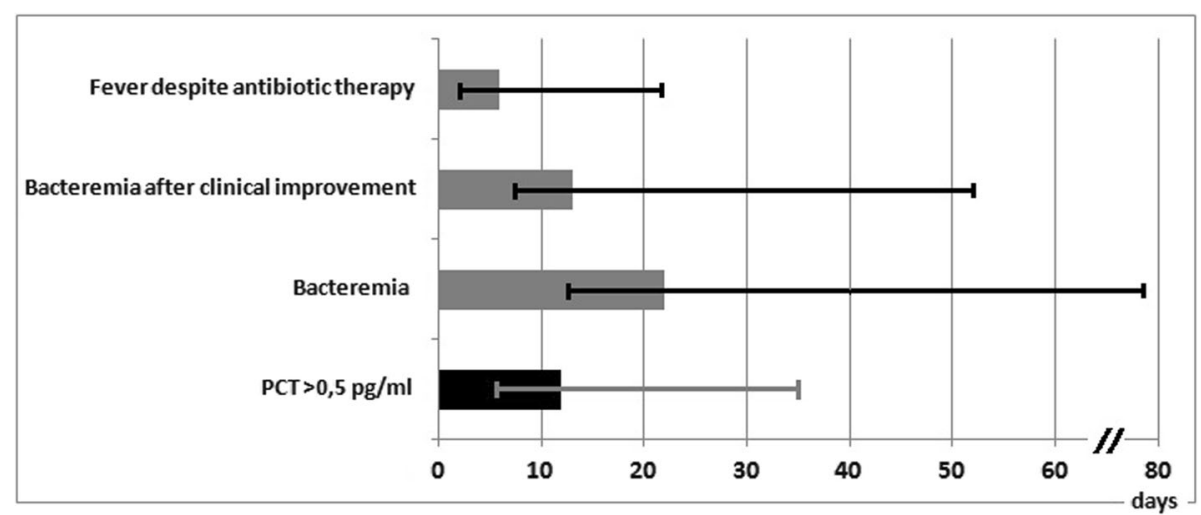

Fig. 1 Clinical and microbiological course of GNB septic thrombophlebitis in critically ill patients. Clinical improvement was defined as withdrawal of inotropic support and fever $<38{ }^{\circ} \mathrm{C}$. The median values (with range) are shown

larger series of cases. Physicians should be aware that serum PCT measurements should be interpreted with caution in assessing the clinical course of GNB endovascular infections. In this setting performance of serial blood cultures seems to remain the most adequate guide to therapy $[1,4]$.

\section{Abbreviations}

CT scan: Computed tomography scan; GNB: Gram-negative bacilli; ICU: Intensive care unit: PCT: Procalcitonin; SAPS II: Simplified Acute Physiology Score

\section{Acknowledgements}

We would like to thank all the participants in this study.

\section{Availability of data and materials}

The datasets used and/or analyzed during the current study are available from the corresponding author on reasonable request.

\section{Authors' contributions}

SAM and FA enrolled patients and collected biosamples and clinical data. MS and SAM performed the statistical analysis for this project, GC created the figure presented. MS, GC, and MV drafted, edited, and finalized the manuscript. All authors read and approved the final manuscript.

\section{Ethics approval and consent to participate}

This study was approved by the Ethics Committee of University of Rome Sapienza RIF/CE 4773. All participants consented prior to enrollment.

\section{Consent for publication}

Not applicable.

\section{Competing interests}

The authors declare that they have no competing interests.

\section{Publisher's Note}

Springer Nature remains neutral with regard to jurisdictional claims in published maps and institutional affiliations.

\section{Author details}

${ }^{1}$ Department of Public Health and Infectious Diseases, University of Rome Sapienza, Viale del Policlinico 155, Rome, Italy. ${ }^{2}$ Azienda Policlinico Umberto I, Rome, Italy. ${ }^{3}$ Department of Anesthesia and Intensive Care Medicine, University of Rome Sapienza, Rome, Italy.
Received: 9 June 2018 Accepted: 3 July 2018

Published online: 23 July 2018

References

1. Thomas-Rüddel DO, Poidinger B, Kott M, Weiss M, Reinhart K, Bloos F, MEDUSA study group. Influence of pathogen and focus of infection on procalcitonin values in sepsis patients with bacteremia or candidemia. Crit Care. 2018;22(1):128.

2. Yan ST, Sun LC, Jia HB, Gao W, Yang JP, Zhang GQ. Procalcitonin levels in bloodstream infections caused by different sources and species of bacteria. Am J Emerg Med. 2017;35(4):579-83.

3. Ayres JS, Schneider DS. Two ways to survive an infection: what resistance and tolerance can teach us about treatments for infectious diseases. Nat Rev Immunol. 2008;8(11):889-95.

4. Ceccarelli G, Giuliano S, Falcone M, Venditti M. Follow-up blood cultures: a 2.0 diagnostic tool in patients with Gram-negative bacteremia and septic thrombophlebitis. Clin Infect Dis. 2018:66(7):1154-5. 